\title{
Domain mapping of periodically poled lithium niobate via terahertz wave form analysis
}

\author{
Y.-S. Lee, ${ }^{\text {a) }}$ T. Meade, M. L. Naudeau, and T. B. Norris \\ Center for Ultrafast Optical Science, University of Michigan, Ann Arbor, Michigan 48109-2099
}

\author{
A. Galvanauskas \\ IMRA America, 1044 Woodridge Avenue, Ann Arbor, Michigan 48105
}

(Received 13 July 2000; accepted for publication 21 August 2000)

\begin{abstract}
We demonstrate a nondestructive probing technique to investigate the inner crystal domain structure of ferroelectric crystals by analyzing the terahertz wave forms generated by optical rectification. Quantitative analysis of the domain structure has been performed for the domain width in periodically poled lithium niobate. Simulation results show that the terahertz wave form analysis reproduces root-mean-square domain width fluctuations. () 2000 American Institute of Physics.
\end{abstract}

[S0003-6951(00)02442-6]

Ferroelectric crystals have been studied and used for many applications including integrated optics, acoustic wave devices, pyroelectric detectors, actuators, and quasiphasematching crystals. These materials consist of domains of uniform polarization below their critical temperature, which are separated by domain walls. Since the domain structure of the materials affects the physical properties of the crystals in many aspects, it is important to understand the patterns of the domain structure. Numerous methods such as chemical etching, ${ }^{1-3}$ x-ray diffraction imaging, ${ }^{4,5}$ second harmonic generation microscopy ${ }^{6-8}$ scanning force microscopy (SFM) ${ }^{9-11}$ electro-optic imaging microscopy (EOIG), ${ }^{12}$ scanning electron microscopy (SEM) ${ }_{13}^{13}$ and near-field scanning optical microscopy (NSOM) $)^{14,15}$ have been used to study ferroelectric domain structures. These techniques have their own merits, but a critical limitation is that they cannot probe the inner crystal domain interface nondestructively. Surface probing techniques like SFM, EOIG, SEM, and NSOM can probe the domain interfaces only on a sample's surface. X-ray diffraction and second harmonic generation acquire two-dimensional images of the domain structure, but these images contain only averaged information of the domain interface along the probe beam direction. One direct method is the observation of differentially etched domains using surface probing tools, although it is unavoidable for the samples to be destroyed.

In this letter, we demonstrate a nondestructive technique for probing the inner crystal domain structure by analyzing terahertz $(\mathrm{THz})$ wave forms generated by optical rectification in ferroelectric crystals. Due to the recent progress of femtosecond lasers, coherent light sources in previously unavailable frequency ranges can be attained through optical nonlinearity of electro-optic and optically nonlinear crystals. Periodic domain structures of ferroelectric materials have been used for second harmonic generation and optical parametric oscillation based on the quasiphasematching technique. Lithium niobate is a widely used ferroelectric material

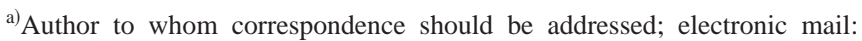
yunslee@eecs.umich.edu
}

because of its large nonlinear optical coefficient. Typically, periodic domain structures are obtained by applying an electric field to a patterned electrode. ${ }^{16}$ Neighboring domains are isolated from the electrodes by a dielectric layer. However, due to the strong coercive electric fields and field leakage out of the electrodes, each domain suffers width fluctuations. ${ }^{2,16}$

Our recent work ${ }^{17,18}$ shows that narrow-band $\mathrm{THz}$ electromagnetic radiation is generated by optical rectification in a periodically poled lithium niobate (PPLN) crystal and that the $\mathrm{THz}$ wave form corresponds to the domain structure of the PPLN. Femtosecond optical pulses are propagated through a PPLN crystal, where the domain width is matched to the walkoff length between the optical and $\mathrm{THz}$ pulses. Thus, the PPLN domain structure can be directly mapped by the $\mathrm{THz}$ wave form. Irregular $\mathrm{THz}$ wave forms due to aperiodic poling can also be easily attained using this technique. Sections of the $\mathrm{THz}$ wave form measured at different time delays are generated at different locations in the PPLN crystal. The resulting $\mathrm{THz}$ wave form maps out the inner crystal domain structure. This $\mathrm{THz}$ imaging technique can be applied to map the three dimensional domain structure of ferroelectric crystals.

The experiment was performed using $800 \mathrm{~nm}, 150 \mathrm{fs}$ pulses from a $250 \mathrm{kHz}$ Ti:sapphire regenerative amplifier, ${ }^{19}$ and an $L=1.2 \mathrm{~mm} z$-cut PPLN crystal with domain length $l_{d}=30 \mu \mathrm{m}$. The sample's height and width are 0.5 and 6.5 $\mathrm{mm}$, respectively. The incident optical pulse energy was 40 $\mathrm{nJ}$, focused to a roughly $100 \mu \mathrm{m}$ spot in the crystal. The radiated $\mathrm{THz}$ was collimated with an off-axis parabola and focused into a 1-mm-thick ZnTe crystal for electro-optic detection of the THz field. ${ }^{20}$ The excitation beam was chopped at $50 \mathrm{kHz}$ for lock-in signal detection. The sample was set in a cryostat which kept the temperature below $20 \mathrm{~K}$ to reduce thermal absorption of the $\mathrm{THz}$ waves inside the material. It has been reported that the $\mathrm{THz}$ absorption can be substantially suppressed at low temperature. ${ }^{21}$ The suppression of the $\mathrm{THz}$ absorption is shown in Fig. 1, where the THz wave forms at (a) $T=19 \mathrm{~K}$ and (b) $298 \mathrm{~K}$ are demonstrated. Each half cycle of the wave form corresponds to a single ferroelectric domain and the $\mathrm{THz}$ wave form at low temperature shows clearly every domain in the PPLN. 


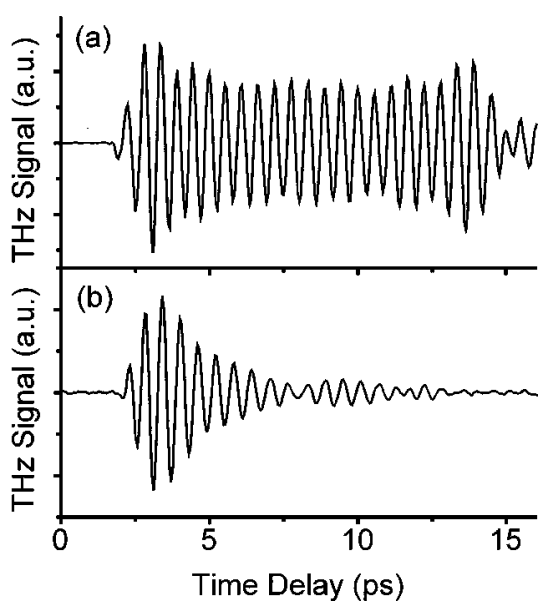

FIG. 1. THz wave form from $1.2 \mathrm{~mm}$ PPLN when the sample temperature is: (a) $T=19 \mathrm{~K}$ and (b) $T=298 \mathrm{~K}$.

Figure 2(a) shows an optical image of the electrode pattern of the PPLN, magnified to exaggerate the domain lengths. The $x$ and $z$ axes are as shown in Fig. 2(a). The optic axis of the sample coincides with the $z$ axis. It is clearly seen that the sample is wedged and the sample surfaces are not perpendicular to the domain interfaces. To probe the entire domain structure of the PPLN, we scan the PPLN sample along the $x$ axis with respect to the fixed excitation beam. The optical excitation beam is vertically positioned in the middle of the sample $(y=0.25 \mathrm{~mm})$. The $\mathrm{THz}$ wave form image of the PPLN domain structure is shown in Fig. 2(b). Fifteen data points are taken along the $x$ axis; values between the data points are interpolated. In the narrower region of the

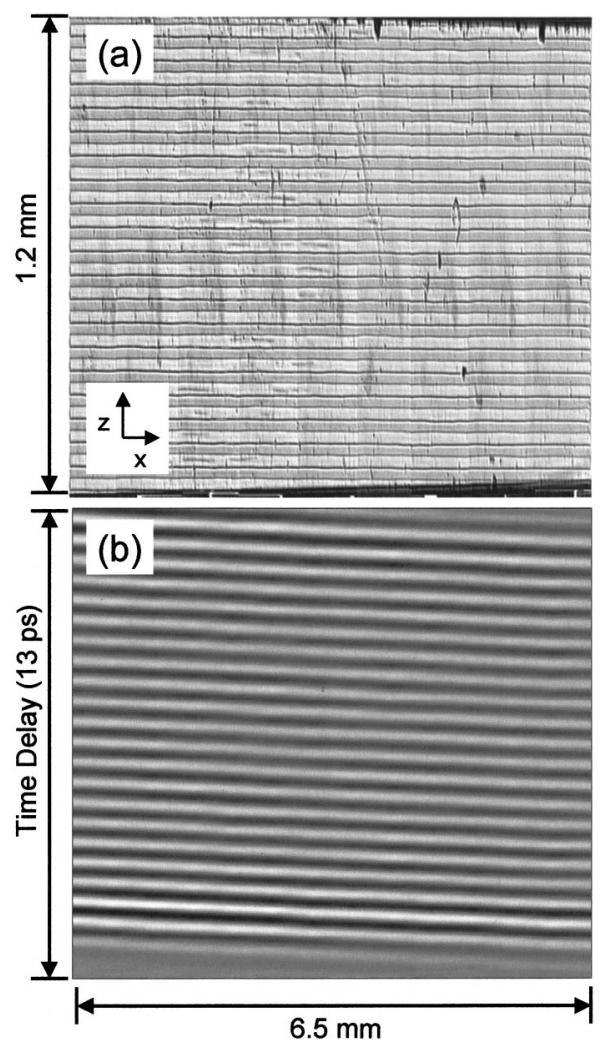

FIG. 2. Two dimensional image of: (a) electrode patterns and (b) THz wave form ( $\mathrm{THz}$ field intensity is proportional to the brightness of the image) of $1.2 \mathrm{~mm}$ PPLN.
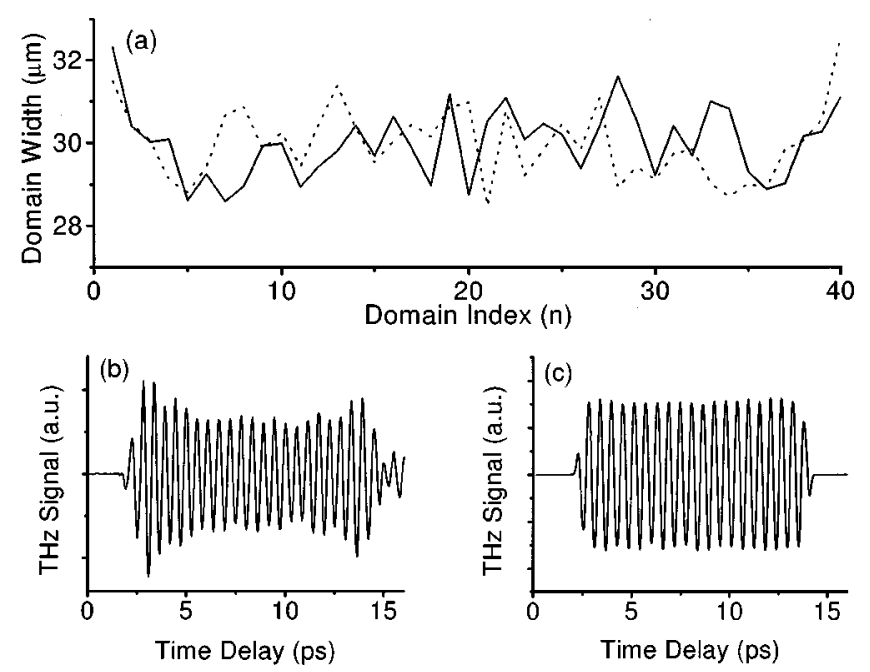

FIG. 3. Domain width fluctuation obtained by zero-crossing positions of $\mathrm{THz}$ wave forms in: (a) experiment (solid lines), and simulation (dotted lines); corresponding $\mathrm{THz}$ wave forms for (b) experiment, and (c) simulation.

crystal, the effective optical beam path is shorter, thus the $\mathrm{THz}$ waves arrive earlier than in the wider region. The slanted domain interfaces to the sample surfaces are also well reproduced in the $\mathrm{THz}$ wave form image. A threedimensional image of the domain structure can be obtained by scanning the sample in the $x-y$ plane.

In order to analyze the domain width fluctuation quantitatively, we interpolate the zero crossing positions of the $\mathrm{THz}$ wave forms using the least-squares method. We calculate the interval between zero crossing positions, and transform the interval to a domain width. The accuracy of the zero crossing method is tested using a numerical calculation of the THz wave form generated in the PPLN structure including domain width fluctuations. The fluctuation of the domain width obtained from zero crossing positions at $x$ $=0.5 \mathrm{~mm}$ is shown as the solid curve in Fig. 3(a), and the corresponding wave form is shown in Fig. 3(b). The average domain width is $30 \mu \mathrm{m}$, with a standard deviation of 0.86 $\mu \mathrm{m}$. It has been reported that the width of the inverted domain is larger than that of the noninverted domain in spite of the strictly regulated electrode pattern, ${ }^{22-24}$ but we did not observe this phenomenon. The difference between the average length of the inverted and noninverted domain is 0.06 $\mu \mathrm{m}$, which is substantially smaller than the standard deviation of the domain width fluctuation $(0.86 \mu \mathrm{m})$. To test how accurately the $\mathrm{THz}$ wave forms can reconstruct the domain structure of the PPLN, we convert the interval of zerocrossing positions into a domain structure with fluctuations. This domain structure is then inserted into a simulation code which solves the wave equation for the $\mathrm{THz}$ field inside the PPLN crystal. ${ }^{17}$ From the zero crossings of the simulated field, we extract a domain structure of the simulated crystal, for self-consistent comparison with our experimental measurements. The simulated wave form is shown in Fig. 3(c), and the domain width fluctuation obtained from the wave form is the dotted curve in Fig. 3(a). As seen in Fig. 3(a), while the detailed correspondence between simulation and experiment is not perfect, the general features of the fluctuations are reproduced very well by the simulation. The stan- 


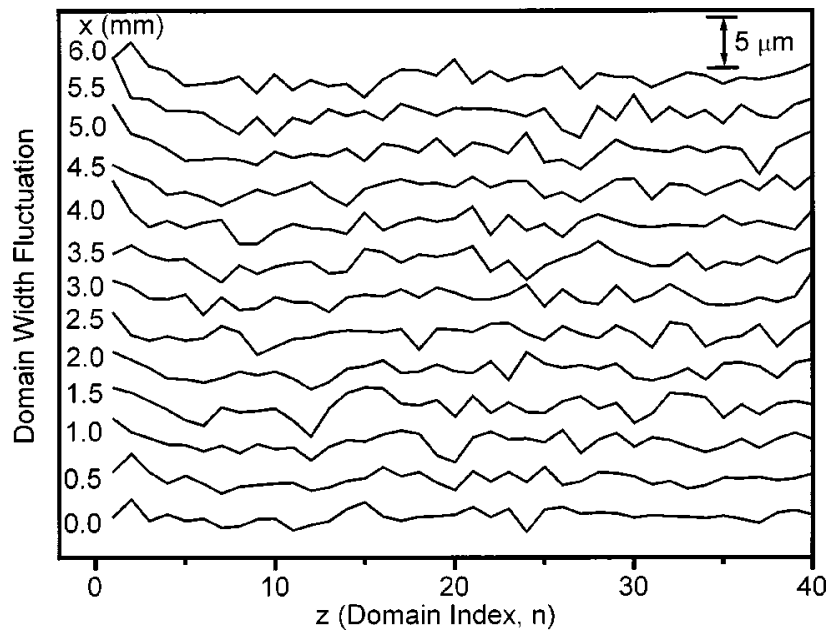

FIG. 4. Domain width fluctuations obtained by zero-crossing positions of $\mathrm{THz}$ wave forms for various sample positions where $x=0.0,0.5,1.0,1.5$, $2.0,2.5,3.0,3.5,4.0,4.5,5.0,5.5$, and $6.0 \mathrm{~mm}$. To see the fluctuations clearly, data sets for the sample positions are offset vertically.

dard deviation of the simulation's result is $0.87 \mu \mathrm{m}$, which is in excellent agreement with the experimental result $(0.86$ $\mu \mathrm{m})$. Our results indicate that this simulation includes the major features necessary to model $\mathrm{THz}$ generation in periodic crystals at low temperature. Further simulation tells us that the zero-crossing method is not appropriate for the quantitative analysis of domain fluctuation when it is more than $5 \%$, although it can still show the qualitative structure variation. In order to do quantitative mapping of irregular structures, deconvolution of the $\mathrm{THz}$ wave form must be performed. Since the $\mathrm{THz}$ wave form of the simulation is the solution of linear wave equation, ${ }^{17}$ the deconvolution should be straightforward.

Figure 4 shows the domain width fluctuations obtained by zero-crossing positions of $\mathrm{THz}$ wave forms at various sample positions. The signal noise is about $0.1 \%$ of the signal amplitude, which is negligible compared to the width fluctuation (3\% of the domain width).

We demonstrate that the inner crystal domain structures of the PPLN can be probed nondestructively by analyzing the THz wave forms generated by optical rectification. Simulation results show that the terahertz wave form analysis can reproduce the domain width fluctuation very well.

The authors wish to thank Gregg Sucha for helpful conversations. This work was supported by ARO under Grant No. DAAH04-96-1-0414 and by NSF under Grant No. DMR-9729109.

${ }^{1}$ A. Sawada and R. Abe, Jpn. J. Appl. Phys. 6, 699 (1967).

${ }^{2}$ V. Ya Shur, E. L. Rumyantsev, E. V. Nikolaeva, E. I. Shishkin, D. V. Fursov, R. G. Batchko, L. A. Eyres, M. M. Fejer, and R. L. Byer, Appl. Phys. Lett. 76, 143 (2000).

${ }^{3}$ S. O. Fregatov and A. B. Sherman, Tech. Phys. Lett. 23, 438 (1997).

${ }^{4}$ P. Rejmánková-Pernot, P. Cloetens, J. Baruchel, J.-P. Guigay, and P. Moretti, Phys. Rev. Lett. 81, 3435 (1998).

${ }^{5}$ Z. W. Hu, P. A. Thomas, and W. P. Risk, Phys. Rev. B 59, 14259 (1999).

${ }^{6}$ S. I. Bozhevolnyi, J. M. Hvam, K. Pedersen, F. Laurell, H. Karlsson, T. Skettrup, and M. Belmonte, Appl. Phys. Lett. 73, 1814 (1998).

${ }^{7}$ Y. Uesu, S. Kurimura, and Y. Yamamoto, Appl. Phys. Lett. 66, 2165 (1995).

${ }^{8}$ S. Kurimura and Y. Uesu, J. Appl. Phys. 81, 369 (1997).

${ }^{9}$ C. Gao, F. Duewer, Y. Lu, and X.-D. Xiang, Appl. Phys. Lett. 73, 1146 (1998).

${ }^{10}$ L. M. Eng, H.-J. Güntherodt, G. Rosenman, A. Skliar, M. Oron, M. Katz, and D. Eger, J. Appl. Phys. 83, 5973 (1998).

${ }^{11}$ A. Gruverman, O. Auciello, and H. Tokumoto, J. Vac. Sci. Technol. B 14, 602 (1996).

${ }^{12}$ V. Gopalan and T. E. Mitchell, J. Appl. Phys. 85, 2304 (1999).

${ }^{13}$ S. Zhu and W. Cao, Phys. Rev. Lett. 79, 2558 (1997).

${ }^{14}$ T. J. Yang, U. Mohideen, and M. C. Gupta, Appl. Phys. Lett. 71, 1960 (1997).

${ }^{15}$ C. Hubert and J. Levy, Appl. Phys. Lett. 73, 3229 (1998).

${ }^{16}$ G. Rosenman, Kh. Garb, A. Skliar, M. Oron, D. Eger, and M. Katz, Appl. Phys. Lett. 73, 865 (1998).

${ }^{17}$ Y.-S. Lee, T. Meade, V. Perlin, H. Winful, T. B. Norris, and A. Galvanauskas, Appl. Phys. Lett. 76, 2505 (2000).

${ }^{18}$ Y.-S. Lee, T. Meade, M. DeCamp, T. B. Norris, and A. Galvanauskas, Appl. Phys. Lett. 77, 1244 (2000).

${ }^{19}$ T. B. Norris, Opt. Lett. 17, 1009 (1992).

${ }^{20}$ Q. Wu and X.-C. Zhang, Appl. Phys. Lett. 68, 1604 (1996).

${ }^{21}$ J. Shikata, M. Sato, T. Taniuchi, H. Ito, and K. Kawase, Opt. Lett. 24, 202 (1999)

${ }^{22}$ K. Kintaka, M. Fujimura, T. Suhara, and H. Nishira, J. Lightwave Technol. 14, 462 (1996).

${ }^{23}$ L. Goldberg, R. W. McElhanon, and W. K. Burns, Electron. Lett. 31, 1576 (1995).

${ }^{24}$ V. Prunery, R. Koch, P. G. Kazansky, W. A. Clarkson, P. St. J. Russel, and D. C. Hanna, Opt. Lett. 20, 2375 (1995). 